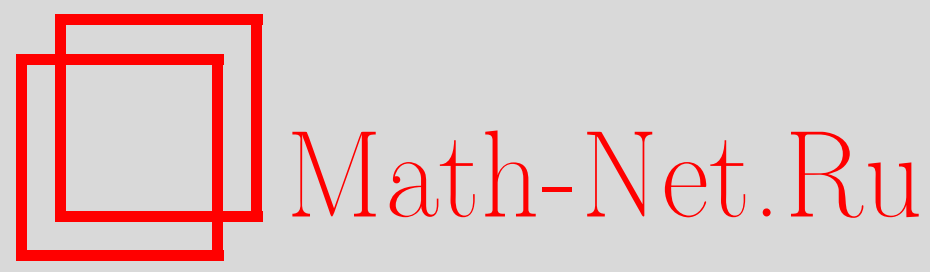

Б. С. Кругликов, Точная классификация невырожденных бездивергентных векторных полей на поверхностях малого рода, Матем. заметки, 1999, том 65, выпуск 3, 336-353

DOI: https://doi.org/10.4213/mzm1057

Использование Общероссийского математического портала Math-Net.Ru подразумевает, что вы прочитали и согласны с пользовательским соглашением http://www.mathnet.ru/rus/agreement

Параметры загрузки:

IP: 54.162 .27 .143

26 апреля 2023 г., 13:50:29

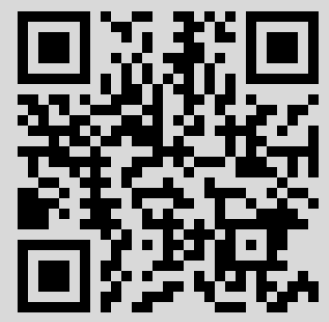




\title{
ТОЧНАЯ КЛАССИФИКАЦИЯ НЕВЫРОЖДЕННЫХ БЕЗДИВЕРГЕНТНЫХ ВЕКТОРНЫХ ПОЛЕЙ НА ПОВЕРХНОСТЯХ МАЛОГО РОДА
}

\section{Б. С. Кругликов}

\begin{abstract}
В работе приводится классификация с точностью до сопряжения в категории $C^{k}$, $k=1, \ldots, \infty$, полных невырожденных бездивергентных векторных полей на компактной поверхности, возможно с краем, почти все траектории которых замкнуты. Дается классификация с точностью до сопряжения произвольных невырожденных бездивергентных полей на поверхностях $S^{2}, \mathbb{R} P^{2}, K^{2}, T^{2}, K^{2} \# \mathbb{R} P^{2}$, возможно с дырками.

Библиография: 11 названий.
\end{abstract}

Введение. Рассмотрим связное компактное двумерное многообразие $P^{2}$ и гладкую меру $\mu=\left|d^{2} v\right|$ на нем. Векторное поле $v$ назьвается бездивергентным, если порожденньй им поток сохраняет меру $\mu$. Всюду ниже мы будем считать, что векторные поля касаются края поверхности $P^{2}$, т.е. определен сдвиг вдоль траекторий за любое время. Локально мера $\mu$ может быть представлена 2-формой $\omega=d^{2} v$, поэтому бездивергентное поле $v$ является локально гамильтоновым, $v=\operatorname{sgrad}_{\omega} F$, т.е. $d F=\omega(*, v)$ для некоторых $F, \omega$. В п. 1-4 мы приводим точную классификацию (т.е. с точностью до сопряжения) в категории $C^{k}, k=1, \ldots, \infty$, невырожденных бездивергентных векторных полей на поверхности $P^{2}$, почти все траектории которых замкнуты. В локальной записи $v=\operatorname{sgrad}_{\omega} F$ невырожденность эквивалентна морсовости функции $F$. В п. $3,5,6$ мы решаем задачу классификации с точностью до сопряжения невырожденных бездивергентных векторных полей на сфере, проективной плоскости, бутылке Клейна, торе и торе с вклеенньм листом Мёбиуса с некоторым (возможно нулевым) количеством выброшенных дисков.

Бездивергентные поля двойственны относительно меры к замкнутым 1-формам, топология и динамика которых активно изучались (см. [1], [2]). Траекторное поведение бездивергентных полей на замкнутых поверхностях эйлеровой характеристики $\chi \leqslant-2$ достаточно сложное. Однако для $\chi>-2$ можно дать точную классификацию.

Если многообразие $P^{2}$ ориентируемо, то выбор ориентации равносилен представлению меры формой объема (симплектической формой) $\mu=\omega$. Поле $v$ становится гамильтоновым. Точная классификация гамильтоновых полей проведена в работах [3], [4]. Эта задача возникла в [5] при редукции типа Пуанкаре задачи о траекторной классификации интегрируемых гамильтоновых систем с двумя степенями свободы, где она решена в $C^{0}$-категории. Мы допускаем разнообразные сепаратрисные связки у рассматриваемых полей, так как соответствующие особенности слоений - типичное явление в интегрируемой гамильтоновой механике с двумя степенями свободы [6]. 
Задаче точной классификации гамильтоновых векторных полей на поверхности $P^{2}$ двойственна задача о классификации троек $\left(P^{2}, \omega, \mathscr{L}\right)$, где $\omega$ - форма объема на поверхности $P^{2}$, а $\mathscr{L}$ - слоение на линии уровня некоторой функции Морса $F$. Для простых функций $F$ классификация проведена в работе [7], для общего случая - в [3]. Аналогично, исходя из классификации бездивергентных векторных полей, можно решить задачу о классификации троек $\left(P^{2}, \mu, \mathscr{L}\right)$, где $\mu$ - гладкая мера на компактной поверхности $P^{2}$, а $\mathscr{L}$ - морсовски невырожденное слоение.

\section{1. Редукция задачи классификации для бездивергентных векторных по-} лей, почти все траектории которых замкнуты. В п. 1-3 мы рассматриваем векторные поля, почти все траектории которых, кроме конечного числа особых, являются замкнутыми. Мы предполагаем наличие хотя бы одного особого слоя слоения поверхности $P^{2}$ траекториями поля $v$, оставляя исключительные случаи до п. 4 . На кольцах и листах Мёбиуса, остающихся после выбрасьвания особых слоев слоения поверхности $P^{2}$ траекториями поля $v$ и слоеных на окружности этими траекториями, имеется естественньй инвариант - класс сопряженности функции периодов потока, т.е. эти функции рассматриваются на ребре - факторпроекции кольца (листа) Мёбиуса по слоению без параметризации.

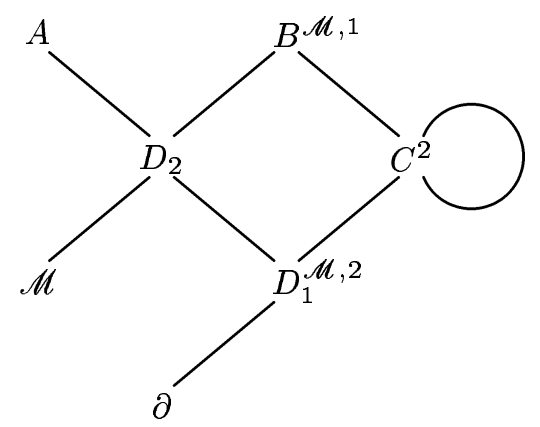

Рис. 1

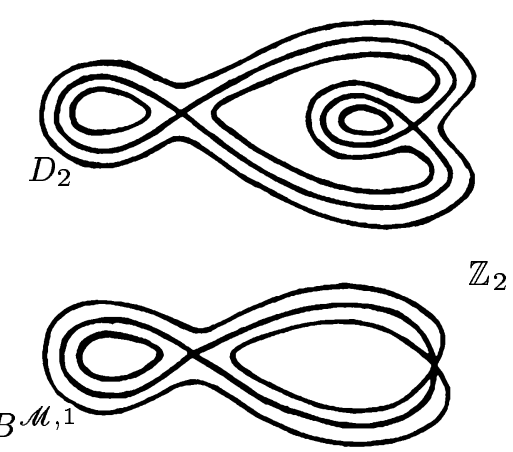

Рис. 2

Рассмотрим факторграф Г поверхности $P^{2}$ по слоению на траектории поля $v$ (рис. 1 ). Ребра графа $Г$ соответствуют расслоенным траекториями кольцам и листам Мёбиуса. Поставим на каждом из них метку $\rho_{k}-$ класс $C^{k}$-сопряженности функции периодов. Вершины графа $Г$, инцидентные одному ребру, соответствуют особьм слоям слоения: $A$ - для эллиптической особой точки, $\partial$ - для граничной компоненты и $\mathscr{M}$ - для центральной окружности расслоенного листа Мёбиуса. Особые слои с ориентируемой окрестностью назьваются буквами-атомами и обозначаются заглавньми латинскими буквами. Таблица букв-атомов малого веса составлена в [6]. Для особых слоев с неориентируемой окрестностью будем использовать заглавные латинские буквы с пометкой $\mathscr{M}$ в индексе (рис. 2). Для вершин $A, \partial$ и $\mathscr{M} \rho$-инвариант полностью классифицирует росток системы $v$. Заметим, что наличие дырок - граничных окружностей - на поверхности не усложняет классификации. Мы всегда можем заклеить дырку расслоенным на окружности диском с эллиптической особой точкой. Это равносильно замене буквы $\partial$ буквой $A$. Итак, точная классификация бездивергентных полей $v$ сведена к классификации ростков локально гамильтоновых векторных полей на гиперболическом особом слое слоения. 
2. Решение редуцированной задачи. В п. 2.1 мы рассмотрим классификацию бездивергентного поля на ростке ориентируемой окрестности особого слоя [3], а в п. 2.2 на ростке неориентируемой окрестности. Мы определяем инварианты при помощи пары $(F, \omega)$, но они не зависят от выбора этой представляющей пары для $v$.

2.1. Ориентируемый случай. Рассмотрим связную компактную ориентируемую поверхность $P^{2}$ с краем $\partial P^{2}=\cup S^{1}$, на котором задана функция Морса (гамильтониан) $F$ с единственным гиперболическим критическим значением, которое мы будем считать нулем. Функция $F$ постоянна на граничных окружностях. Пусть $\left.F\right|_{\partial P^{2}}= \pm 1$. Таким образом, поверхность $P^{2}$ содержит в качестве деформационного ретракта связньй граф $K=F^{-1}(0)$ с вершинами кратности 4 . Всеграничные кольца $P^{2} \backslash K=\sqcup C_{j}$ распадаются на положительные $(F>0)$ и отрицательные $(F<0)$, причем к каждому ребру графа $K$ примыкают разнозначные кольца. Поверхность $P^{2}$ с перечисленными свойствами назьвается буквой-атомом, а окрестности компонент границы - ее кониами. Рассмотрим на $P^{2}$ симплектическую форму $\omega$ и соответствующее гамильтоново поле $\operatorname{sgrad}_{\omega} F$. Любое невырожденное гамильтоново поле $v$, касающееся слоения $\mathscr{I}=\{F=$ const $\}$, представимо в виде $v=\operatorname{sgrad}_{\omega} F$ для некоторой пары $(F, \omega)$. Редуцированная задача состоит в классификации ростков гамильтоновых векторных полей $v$ на графе $K \subset P^{2}$. Переходим к описанию инвариантов.

Рассмотрим произвольную вершину $V$ графа $K$. В ее окрестности существуют [8] локальные координаты $(x, y)$ такие, что $F=x y$ и $\omega=\Lambda(F) d x \wedge d y, \Lambda(0)>0$. В малой окрестности вершины граф $K$ есть “крестик". Трансверсали к его сторонам являются локально трансверсалями потока. Дополнение к графу локально состоит из четырех компонент.

Лемма 1 (см. [5]). Время пробегания потока $\operatorname{sgrad}_{\omega} F$ около вериины $V$ равно $-\Lambda_{V}(F) \ln |F|+c(F)$, әде $\Lambda_{V}$ ис- гладкие функиии на полуинтервале $[0, \varepsilon)$.

Слагаемые $-\Lambda_{V}(F) \ln |F|$ и $c(F)$ назьваются соответственно бесконечной и конечной частями функиии периода (они определены не однозначно). Если мы разложим функцию $\Lambda_{V}(F)$ в ряд Тейлора $\sum_{i=0}^{\infty} \lambda_{V, i} F^{i}$, то получим систему инвариантов автосимплектоморфизма $\Phi: \Lambda_{V, k}(t)=\sum_{i=0}^{k} \lambda_{V, i} t^{i}$. Из леммы следует, что для конца $i$ буквы-атома ( $i$-й компоненты границы $\partial P^{2}-$ кольца $\left.C_{i}\right)$ для функции периодов векторного поля $v$ на ребре имеет место асимптотическое равенство

$$
\Pi_{\omega, i}(F)=-\sum_{j=1}^{m_{i}} \Lambda_{V_{i_{j}}, k}(F) \ln |F|+c_{i}^{k}(F)
$$

где $\Lambda_{V_{i_{j}}, k}(F)=\sum_{r=0}^{k} \lambda_{V_{i_{j}}, r} F^{r}-\Lambda$-инварианты для $m_{i}$ вершин $V_{i_{j}}$ графа $K$, лежаших на границе кольца $C_{i}$, а $c_{i}^{k} \in C^{k}[0, \varepsilon)$. В случае $k=\infty$ мы берем в качестве $\Lambda_{V_{i_{j}}, k}(F)$ любую гладкую функцию с рядом Тейлора $\sum_{r=0}^{\infty} \lambda_{V_{i_{j}}, r} F^{r}$.

ПРЕДЛОЖЕНИЕ 1 [3]. Для любого конца $і$ буквы-атома $\left(P^{2}, K\right)$ существует функиия Морса $F$ вблизи графа $K$ такая, что слоение на связные компоненты уровня $\{F=$ const $\}$ совпадает со слоением $\mathscr{I}$, и конечная часть функиии периодов $c_{i}^{k}$ в разложсении (1) равна 0. Эта функиия определена с точностью до прибавления 
функции $f$, постоянной на листах слоения $\mathscr{I}$, такой, что ее $k$-струя на графе $K$ равна 0 .

Так как $H^{2}\left(P^{2}\right)=H^{2}(K)=0$, мы можем записать $\omega=d \alpha$, где $\left.\alpha\right|_{K} \equiv 0$.

Рассмотрим для каждого кольца $C_{j} \subset P^{2} \backslash K$ сечение $\beta_{j}$ потока векторного поля $v=\operatorname{sgrad}_{\omega} F$, трансверсально входящее в ребро графа $K$. Фиксируем разрез $s_{m}-$ сечение потока, трансверсально пересекающее ребро $e_{m}$. Рассмотрим кольцо $C_{i}$, для определенности положительное $C_{i}^{+}$, т.е. $\left.F\right|_{C_{i}}>0$. На нем есть сечение $\beta_{i}$ и полуразрезы $s_{1 i}^{+}, \ldots, s_{m_{i} i}^{+}$. Обозначим промежуток вдоль поля $\operatorname{sgrad}_{\omega} F$ между $s_{j i}^{+}$и $s_{j+1, i}^{+}$через $U_{j i}=\left[s_{j i}^{+}, s_{j+1, i}^{+}\right) \subset \bar{C}_{i}^{+}, \bar{C}_{i}^{+}=\bigsqcup_{j} U_{j i}$. Пусть для определенности $\beta_{i} \subset U_{1 i}, \beta_{i} \cap K=p_{i}$, и точка $V_{i_{j}}$ лежит на границе $U_{j i}$. Определим функцию $\varkappa: \bar{C}_{i}^{+} \rightarrow \mathbb{R}$ следующим обраउом:

$$
\begin{gathered}
\varkappa=0 \text { на } K \cap \bar{C}_{i}^{+},\left.\quad \varkappa\right|_{\beta_{i}}=g_{i}, \quad g_{i} \in C^{\infty}\left(\beta_{i}\right), \quad g_{i}\left(p_{i}\right)=0, \\
\varkappa(x)=\int_{l} \alpha+g_{i}\left(x_{0}\right) \text { на } U_{1 i},
\end{gathered}
$$

где $l$ - отрезок интегральной траектории поля $\operatorname{sgrad}_{\omega} F$, лежащий в $U_{1 i}$ и соединяющий точку $x$ с точкой $x_{0}$ на сечении $\beta_{i}$. Далее, для $x_{0} \in s_{2 i}^{+}$определим

$$
\varkappa\left(x_{0}\right)=\lim _{\substack{x \rightarrow x_{0} \\ x \in U_{1 i}}} \varkappa(x)+\Lambda_{V_{i_{1}}, k}\left(F\left(x_{0}\right)\right) \ln F\left(x_{0}\right)-\frac{1}{m_{i}} c_{i}^{k}\left(F\left(x_{0}\right)\right),
$$

а для $x_{1} \in U_{2 i}$

$$
\varkappa\left(x_{1}\right)=\varkappa\left(x_{0}\right)+\int_{l} \alpha,
$$

где $l$ - отрезок траектории поля $\operatorname{sgrad}_{\omega} F$ в $U_{2 i}$, соединяющий точки $x_{1}$ и $x_{0} \in s_{2 i}^{+}$. Для $x_{0} \in s_{3 i}^{+}$определим

$$
\varkappa\left(x_{0}\right)=\lim _{\substack{x \rightarrow x_{0} \\ x \in U_{2 i}}} \varkappa(x)+\Lambda_{V_{i_{2}}, k}\left(F\left(x_{0}\right)\right) \ln F\left(x_{0}\right)-\frac{1}{m_{i}} c_{i}^{k}\left(F\left(x_{0}\right)\right)
$$

и т.д. Если мы сделаем полньй оборот и снова определим $\left.\varkappa\right|_{U_{1 i}}$, то получим то же значение.

Проделаем эту процедуру для всех колец. Разложим $\varkappa$ в ряд Тейлора по $F$ на $s_{m}^{ \pm}$в окрестности точки $s_{m}^{0}$, где $s_{m}^{ \pm}$- часть разреза $s_{m}$, соответствующая знаку “土” функции $F$, а точка $s_{m}^{0}$ соответствует пересечению ребра $e_{m}$ :

$$
\varkappa^{ \pm}=\sum_{i=1}^{k+1} \delta_{i}^{ \pm} F^{i}+o\left(|F|^{k+1}\right), \quad k<\infty, \quad \text { или } \quad \varkappa^{ \pm} \doteq \sum_{i=1}^{\infty} \delta_{i}^{ \pm} F^{i}, \quad k=\infty .
$$

Обозначим через $\delta_{k}^{m}(t)$ сумму $\sum_{i=1}^{k+1}\left(\delta_{i}^{+}-\delta_{i}^{-}\right) t^{i-1}$, где $m$ нумерует ребра. Заклеим дисками граничные компоненты поверхности $P^{2}$. Получим замкнутую ориентируемую поверхность $\widetilde{P}^{2}$. Рассмотрим стандартное клеточное разбиение $\widetilde{P}^{2}: 0$-клетки - вершины $K, 1$-клетки - ребра и 2-клетки - приклеиваемые диски. Пусть $\mathbb{R}_{k}[t]-$ множество полиномов (рядов) $\{p(t) \mid \operatorname{deg} p \leqslant k\}$. 
1-цепь $\delta_{k}(\omega)=\sum_{m} \delta_{k}^{m}(t) e_{m} \in C_{1}\left(\widetilde{P}^{2} ; \mathbb{R}_{k}[t]\right)$ определена неоднозначно. При изменении разрезов $s$, сечений $\beta$ и начальных значений $g$ функции $\varkappa$ на сечениях 1 -цепь $\delta_{k}(\omega)$ изменяется на гранищу. Таким образом, мы получаем однозначно определенньй элемент $\left[\delta_{k}(\omega)\right] \in C_{1} / B_{1}\left(\widetilde{P}^{2} ; \mathbb{R}_{k}[t]\right)$. Имеем $C_{1} / B_{1}=C_{1} / Z_{1}+Z_{1} / B_{1}=B_{0}+H_{1}$. Считая 1-цепи $\left\{t^{l} e_{m}\right\}_{l=0, \ldots, k}(m$ нумерует ребра) ортонормированным базисом, мы получаем скалярное произведение в $C_{1}\left(\widetilde{P}^{2} ; \mathbb{R}_{k}[t]\right)$. Мы можем однозначно разложить $\delta_{k}(\omega)=w_{k}(\omega)+$ $v_{k}(\omega)$, где $v_{k}(\omega) \in Z_{1}\left(\widetilde{P}^{2} ; \mathbb{R}_{k}[t]\right)=\left\{c \in C_{1} \mid \partial c=0 \in C_{0}\right\}, w_{k} \perp v_{k}$. Положим

$$
\Delta_{k}^{\prime}(\omega) \stackrel{\text { def }}{=} \partial w_{k}=\partial \delta_{k} \in B_{0}\left(\widetilde{P}^{2} ; \mathbb{R}_{k}[t]\right), \quad Z_{k}(\omega) \stackrel{\text { def }}{=}\left[v_{k}(\omega)\right] \in Z_{1} / B_{1}=H_{1}\left(\widetilde{P}^{2} ; \mathbb{R}_{k}[t]\right) .
$$

Инвариант $\Delta_{k}^{\prime}(\omega)$ является продолжением аналогичного инварианта в $C^{0}$-категории из [5]. В гладкой категории для классификации нам необходимо знать росток функции периодов на графе $K$, который не восстанавливается по $\Delta_{k}^{\prime}(\omega)$ и $\Lambda$. Поэтому мы вводим инвариант $\Delta_{k}(\omega) \in B^{2}\left(\widetilde{P}^{2} ; \mathbb{R}_{k}[t]\right)$, равный $\sum_{i}\left(j_{k} c_{i}^{k}\right) C_{i}$, где $j_{k} c_{i}^{k}-k$-струя конечной части функции периодов $c_{i}^{k}$ на кольце $C_{i}$ (см. (1)). Априори инвариант лежит в $Z^{2}\left(\widetilde{P}^{2}, \mathbb{R}_{k}[t]\right)$, но условие равенства суммы периодов по положительным и отрищательным кольцам показьвает, что $\Delta_{k} \in B^{2}$. Кроме того, существует гомоморфизм $B^{2} \rightarrow B_{0}$ такой, что $\Delta_{k} \mapsto \Delta_{k}^{\prime}$.

Обозначим через $\operatorname{Inv}_{k}\left(P^{2}, v\right)_{i}$ набор инвариантов $\Lambda_{V, k}, Z_{k}$ и $\Delta_{k}$, определенных по задающей слоение $\mathscr{I}$ функции $F$, удовлетворяющей предложению 1 .

Теорема 1 [3]. Ростки гамильтоновых векторных полей $v=\operatorname{sgrad}_{\omega} F$ на графе $K \subset P^{2}$ полностью $C^{k}$-классифицируются набором инвариантов $\operatorname{Inv}_{k}\left(P^{2}, v\right)_{i}$, m.е. два гамильтоновых векторных поля, $v$ на букве-атоме $\left(P^{2}, K\right)$ и $v^{\prime}$ на $\left(P^{2^{\prime}}, K^{\prime}\right)$, точно $C^{k}$-эквивалентны тогда и только тогда, когда соответствующие инварианты $\operatorname{Inv}_{k} u \operatorname{Inv}_{k}^{\prime}$ совпадают.

2.2. Неориентируемый случай. Рассмотрим неориентируемую букву-атом $\left(P^{2}, K\right)$ и бездивергентное векторное поле $v$ на ней. Рассмотрим знаконеопределенную функцию Морса $\pm F$ и знаконеопределенную форму объема $\pm \omega$, представляющую меру $\mu$, такие, что $v=\operatorname{sgrad}_{\omega} F$. Это означает, что мы рассматриваем ориентирующее накрытие $\pi: \widehat{P}^{2} \stackrel{\mathbb{Z}_{2}}{\rightarrow} P^{2}$, поднимаем поле $v$ до поля $\widehat{v}$ на $\widehat{P}^{2}$ и находим функцию Морса $\widehat{F}$ и форму объема $\widehat{\omega}$ на $\widehat{P}^{2}$ такие, что $\widehat{v}=\operatorname{sgrad}_{\widehat{\omega}} \widehat{F}$. Будем считать $\left.F\right|_{K}=0$. Положим $\widehat{K}=\pi^{-1}(K)$. Группа монодромии накрытия инволютивно действует на $\widehat{P}^{2}: x \mapsto \sigma(x)$, причем $\sigma^{*}(\widehat{F})=-\widehat{F}, \sigma^{*} \widehat{\omega}=-\widehat{\omega}$. Мы будем, допуская вольность, говорить о $\widehat{F}$ как о $\pm F$. Определим $\Lambda$-функцию для $\widehat{v}$ и $\widehat{F}$ в окрестности вершины $A \in \widehat{K} \subset \widehat{P}^{2}$ при помощи леммы 1 , причем по определению $j_{\infty} U(0)=0$ для $U(\widehat{F})=\Lambda_{A}(\widehat{F})-\Lambda_{\sigma(A)}(-\widehat{F})$, т.е. $\lambda_{A, 2 i}=\lambda_{\sigma(A), 2 i}, \lambda_{A, 2 i+1}=-\lambda_{\sigma(A), 2 i+1}, i \geqslant 0$.

ПРЕДЛОЖЕНИЕ 2. ДЛя любого конца $і$ буквы-атома $\left(P^{2}, K\right)$ существует функиия Морса $\pm F$ вблизи графа $K$ такая, что слоение на связные компоненты уровня $\{|F|=$ const $\}$ совпадает со слоением $\mathscr{I}$, и конечная часть функции периодов $c_{i}^{k}$ в разлохсении (1) равна $0 ; k$-струя этой функции на графе $K$ определена однозначно.

Для группы цепей накрытия имеем разложение $C_{*}\left(\widehat{P}^{2} ; R\right)=C_{*}^{-}\left(\widehat{P}^{2} ; R\right) \oplus C_{*}^{+}\left(\widehat{P}^{2} ; R\right)$, где $R$ - произвольное кольцо, а $C_{*}^{ \pm}\left(\widehat{P}^{2} ; R\right)=\left\{c \in C_{*}\left(\widehat{P}^{2} ; R\right) \mid \sigma_{*}(c)= \pm c\right\}$. При этом $C_{*}^{+}\left(\widehat{P}^{2} ; R\right) \simeq C_{*}\left(P^{2} ; R\right)$, а $C_{*}\left(P^{2} ; R_{T}\right):=C_{*}^{-}\left(\widehat{P}^{2} ; R\right)$ называются иепями со скрученными коэффиииентами. Циклы, границы и гомологии со скрученными коэффициентами определяются обычным образом. 
Итак, выбирая функцию Морса $\pm F$, как в предложении 2 , мы получаем однозначно определенный инвариант

$$
\begin{gathered}
\Lambda_{k}\left(P^{2}, v\right)=\left\{j_{k} \Lambda_{V}(\widehat{F})(0)\right\}_{V} \in C_{0}\left(P^{2} ; \mathbb{R}_{k, \text { even }}[t]\right) \oplus C_{0}\left(P^{2} ; \mathbb{R}_{k, \text { odd }}[t]_{T}\right), \\
\mathbb{R}_{k, \text { even }}[t]:=\mathbb{R}_{k / 2}\left[t^{2}\right], \quad \mathbb{R}_{k, \text { odd }}=t \mathbb{R}_{k-1, \text { even }}[t], \quad k=1, \ldots, \infty .
\end{gathered}
$$

Перейдем теперь к $\Delta Z$-инварианту. Выберем на $\widehat{P}^{2}$ форму объема $\omega$, индуцирующую естественную ориентацию, такую, что форма $\pm \omega$ представляет меру $\mu$. Так как $H^{2}\left(\widehat{P}^{2}\right)=H^{2}(\widehat{K})=0$, можно записать $\omega=d \alpha$. Поскольку $\sigma^{*} \omega=-\omega$, мы можем считать $\sigma^{*} \alpha=-\alpha$, а также $\left.\alpha\right|_{\widehat{K}} \equiv 0$.

Выберем для каждого кольца из $P^{2} \backslash K$ сечение $\beta$ и для каждого ребра графа $K$ сечение $s$. Поднимем их до $\sigma$-инволютивного множества разрезов и сечений в $\left(\widehat{P}^{2}, \widehat{K}\right)$. Как и в п. 2.1, определим при помощи функции $\varkappa 1$-цепь $\delta_{k}(\omega) \in C_{1}\left(\widetilde{\widehat{P}^{2}} ; \mathbb{R}_{k}[t]\right)$, причем легко видеть, что она удовлетворяет условию $\sigma^{*} \delta_{k}=-\delta_{k}$, т.е. как и ранее, устраняя произвол при определении $\varkappa$, мы имеем элемент $\delta_{k}(\mu)=\left[\delta_{k}(\omega)\right] \in C_{1} / B_{1}\left(\widetilde{P}^{2} ; \mathbb{R}_{k}[t]_{T}\right)$ (это определение корректно, поскольку ${\widetilde{P^{2}}}^{2} \widehat{\widetilde{P}^{2}}$ ). Производя разделение, как и в п. 2.1, получаем инварианты

$$
\begin{gathered}
\Delta_{k}^{\prime}(\mu) \stackrel{\text { def }}{=} \partial \delta_{k} \in B_{0}\left(\widetilde{P}^{2} ; \mathbb{R}_{k}[t]_{T}\right), \quad Z_{k}(\mu) \in H_{1}\left(\widetilde{P}^{2} ; \mathbb{R}_{k}[t]_{T}\right), \\
\Delta_{k}(\mu)=\sum_{i}\left(j_{k} c_{i}^{k}\right) C_{i} \in B^{2}\left(\widetilde{P}^{2}, \mathbb{R}[t]_{T}\right) .
\end{gathered}
$$

Обозначим через $\operatorname{Inv}_{k}\left(P^{2}, v\right)_{i}$ инварианты $\Lambda_{k}\left(P^{2}, v\right), \Delta_{k}\left(P^{2}, v\right)$ и $Z_{k}\left(P^{2}, v\right)$, определенные по функции $\pm F$ из предложения 2 ( $i$ обозначает конец неориентируемого атома $\left.P^{2}\right)$.

Теорема 2. Ростки бездивергентных полей $v$ на графе $K \subset P^{2}$ полностью $C^{k}$-классифицируются набором инвариантов $\operatorname{Inv}_{k}\left(P^{2}, v\right)_{i}$ : два бездивергентных поля, $v$ на букве-атоме $\left(P^{2}, K\right)$ и $v^{\prime}$ на $\left(P^{2}{ }^{\prime}, K^{\prime}\right)$, точно $C^{k}$-эквивалентны тогда и только тогда, когда соответствующие инварианты $\operatorname{Inv}_{k} u \operatorname{Inv}_{k}^{\prime}$ совпадают.

Доказательство проводится с помощью гомотопического метода, как и в $[3, \S 2,3]$.

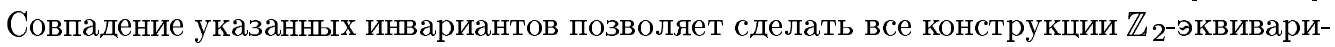
антными относительно действия обращающей ориентацию инволюции $\sigma($ в $[3, \S 6]$ рассматривалась другая инволюция, сохраняющая ориентацию и имеющая неподвижные точки).

3. Классификационная теорема для резонансных бездивергентных векторных полей. Векторные поля на сфере, проективной плоскости и бутылке Клейна.

ТЕОРема 3. а) Два невырожсденных бездивергентных векторных поля, $v$ на компактной поверхности $P^{2}$ и $v^{\prime}$ на $P^{2}{ }^{\prime} \simeq P^{2}, C^{k}$-әквивалентны тогда и только тогда, когда два соответствуюших графа Г и $\Gamma^{\prime}$, снабжсеных метками-инвариантами $\rho_{k}$ на сторонах и метками-инвариантами $\operatorname{Inv}_{k}$ на каждой вериине, отличной от $A, \mathscr{M}$ и $\partial$, изоморфны. Это означает существование изоморфизма $\Gamma \stackrel{\sim}{\rightarrow} \Gamma^{\prime}$ и выбора концов $i$ (т.е. инцидентных ребер, фигурируюших в предлохсениях 1,2 ) гиперболических вериин графов $Г$ и $\Gamma^{\prime}$ таких, что все инварианты сохраняются при изоморфизме и отмеченные концы переходят в отмеченные. 
б) Тем самым получена классификация с точностью до сопряжения невырожденных бездивергентных векторных полей на сфере, проективной плоскости и бутылке Клейна с дырками (выброшенными дисками) $P^{2} \backslash \bigsqcup_{j=1}^{k} D_{j}^{2}, k \geqslant 0, P^{2}=S^{2}$, $\mathbb{R} P^{2}$ или $K^{2}$.

4. Бездивергентные векторные поля без особых точек. В этом пункте мы классифицируем с точностью до сопряженности бездивергентные поля $v$ на $P^{2}$ без особых точек. Существование такого поля на $P^{2}$ накладьвает ограничения на эйлерову характеристику: $\chi\left(P^{2}\right)=0$. Следовательно, $P^{2} \simeq S^{1} \times I, S^{1} \widetilde{\times} I, T^{2}, K^{2}$. В первых двух случаях (для кольца и листа Мёбиуса) слоение на траектории - стандартное слоение на окружности: $S^{1} \times I \rightarrow I=(-1,1), S^{1} \widetilde{\times} I \rightarrow[0,1)$. Поэтому в этих случаях полным инвариантом является функция периодов (точнее, ее класс $C^{k}$-сопряженности; в дальнейшем мы не будем их различать) $\Pi:(-1,1) \rightarrow \mathbb{R}$ или $\Pi:[0,1) \rightarrow \mathbb{R}$. Заметим, что во втором случае функция П является разрывной в нуле:

$$
\lim _{t \rightarrow+0} \Pi(t)=2 \Pi(0) .
$$

Рассмотрим случай $P^{2}=K^{2}$. Для любой точки $x$ существует окрестность с координатами $(p, q)$ такая, что в них форма $\omega$, представляющая меру $\mu=|\omega|$, и векторное поле $v$ записьваются в стандартном виде: $\omega=d p \wedge d q, v=\partial_{p}$. Координаты $(p, q)$ определены с точностью до замен $(p, q) \mapsto(p+Q(q), q+$ const $)$, а при смене ориентации $\omega \mapsto-\omega$ добавляется замена $(p, q) \mapsto(p$, const $-q)$. Поэтому на ориентированной локальной трансверсали имеется параметризация, определенная с точностью до сдвигов. Легко видеть, что существует замкнутая трансверсаль $u: S^{1} \rightarrow K^{2}$ потока $v$. Фиксируя ориентацию, получаем циклический параметр $q(\bmod T)$ на $u\left(S^{1}\right)$. Рассуждения, приведенные выше, показьвают, что отображение последования $u\left(S^{1}\right) \rightarrow u\left(S^{1}\right)$ имеет вид $q \mapsto q_{0}-q$. Следовательно, все траектории поля $v$ замкнуты, и единственным инвариантом является функция периодов $\Pi:[0,1] \rightarrow \mathbb{R}$. Здесь функция П является разрывной на каждом конце, причем характер разрьва такой же, как и в случае листа Мёбиуса $\mathscr{M}^{2}=S^{1} \widetilde{\times} I$, рассмотренном вьше, поскольку $K^{2}=\mathscr{M}^{2} \cup \mathscr{M}^{2}$.

Пусть теперь $P^{2} \simeq T^{2}$. Если число вращения векторного поля $v$ рационально, то существует замкнутая траектория, а потому все траектории замкнуты, и единственным инвариантом является функция периодов $\Pi: S^{1} \rightarrow \mathbb{R}$. Пусть число вращения иррационально. Выберем базис циклов $\gamma_{i}$ и координаты $\varphi^{i}(\bmod 1)$ на торе $T^{2} ; \gamma_{i}=\left\{\varphi^{i+1}=0\right\}$. Из бездивергентности следует, что индекс такого поля вдоль базисного цикла равен 0. Если обмотка тора иррациональная, т.е. нет замкнутых траекторий, то, пользуясь стандартной техникой уничтожения особенностей (см., например, $[1$, рис. 4]), можем продеформировать базисные циклы $\gamma_{i}$ в трансверсали потока. Будем считать их продеформированными. Как было показано вьше для $K^{2}$, на трансверсали имеется естественная параметризация $x(\bmod T)$; нормируя ее, получаем канонические координаты $\varphi^{i}(\bmod 1)$ на циклах. Пусть число вращения в этих циклах равно $\lambda$. Это число определено по модулю дробно-линейных преобразований с матрицей $A \in \mathrm{Gl}_{2}(\mathbb{Z})$.

Определим теперь "среднюю длину" $v_{0}$ вектора $v$. Для точки $z_{0} \in T^{2}$ и числа $t \in \mathbb{R}_{+}$ обозначим через $\tau_{1}(t)$ (через $\tau_{2}(t)$ ) число пересечений вьпущенной из $z_{0}$ траектории с параллелью $\gamma_{1}\left(\right.$ с меридианом $\left.\gamma_{2}\right)$ за время $t$. Положим

$$
v_{0}=\lim _{t \rightarrow \infty} \frac{\tau_{1}(t)}{t \sin \alpha}=\lim _{t \rightarrow \infty} \frac{\tau_{2}(t)}{t \cos \alpha},
$$


где $\alpha$ - угол вращения, связанньй с числом вращения формулой $\lambda=\operatorname{tg} \alpha$. В бескоординатных обозначениях пара $\left(\lambda, v_{0}\right)$ представляется инвариантом $S=v_{0} \cos \alpha\left[\widehat{\gamma_{2}}\right]-$ $v_{0} \sin \alpha \widehat{\left[\gamma_{1}\right]} \in H^{1}\left(T^{2} ; \mathbb{R}\right)$, где $\widehat{\left[\gamma_{1}\right]}, \widehat{\left[\gamma_{2}\right]}$ - двойственньй когомологический базис к $\left[\gamma_{1}\right]$, $\left[\gamma_{2}\right] \in H_{1}\left(T^{2} ; \mathbb{R}\right)$. Кроме того,

$$
S=\frac{1}{|\omega|}\left[i_{v} \omega\right] \in H^{1}, \quad|\omega|=\oint_{T^{2}} \omega .
$$

Замкнутость формы $i_{v} \omega$ следует из бездивергентности $v, \mathscr{L}_{v} \omega=0$.

ТЕОРемА 4. Для почти всех иррациональных чисел вращения $\lambda$ системы $v$ на торе $T^{2}$ классифицируются инвариантом $S$.

ДокАЗАТЕльство. Мы определили на параллели $\gamma_{1}$ параметр $x(\bmod 1)$ такой, что отображение последования имеет вид $x \mapsto x+\theta, \theta=1 / \lambda$, причем величина $1 / \lambda(\bmod 1)$ не зависит от выбора меридиана $\gamma_{2}$. Пусть $w(x)$ - время пробегания векторньм полем $v$ расстояния от точки $x$ на параллели до первого пересечения $x+\theta$. Если $v$-стандартное линейное поле на торе, то для стандартной параллели функция $w(x)=1 /\left(v_{0} \sin \alpha\right)$ постоянна. Для того чтобы система $\left(T^{2}, v\right)$ была эквивалентна линейной, необходимо и достаточно существование новой параллели $\widetilde{\gamma}_{1}$, для которой функция $\widetilde{w}(x)$ постоянна. При изменении параллели функция $w(x)$ заменяется на $w(x)+f(x)-f(x+\theta)$. Мы хотим найти $C^{k}$-гладкую функцию $f$ на $S^{1}$ такую, что $w(x)+f(x)-f(x+\theta)=1 /\left(v_{0} \sin \alpha\right)$. Положим $g(x)=w(x)-1 /\left(v_{0} \sin \alpha\right)$. Очевидно, что $\oint g(x) d x=0$, т.е. необходимое условие для разрешимости уравнения $g(x)+f(x)-f(x+\theta)=0$ вьполнено.

Итак, построение изоморфизма векторных полей сведено к нахождению $\left(C^{k}\right.$-)решения уравнения $f(x+\theta)-f(x)=g(x)$. Это уравнение называется гомологическим и возникает при приведении диффеоморфизма окружности к повороту (аналитический случай был рассмотрен В.И. Арнольдом [9], а гладкий - Ю. Мозером [10]). Иррациональное число $\eta$ называется числом тuna $(K, \sigma), K, \sigma>0$, если для любых целых $p$ и $q \neq 0$ выполнено неравенство

$$
\left|\eta-\frac{p}{q}\right| \geqslant \frac{K}{|q|^{2+\sigma}} .
$$

Почти любое вещественное число является числом типа $(K, \sigma)$ для некоторой такой пары. Пусть $\theta$ является числом типа $(K, \sigma)$ (это свойство не зависит от выбора базиса). Тогда гомологическое уравнение с любой гладкой правой частью и с нулевым средним значением имеет гладкое решение.

Если иррациональное число $\theta$ не является числом типа $(K, \sigma)$, то гомологическое уравнение может не иметь гладкого решения. Для таких $\theta$ существуют неэквивалентные бездивергентные векторные поля без особенностей на торе с совпадающими $S$-инвариантами. В этом случае необходимо требовать $C^{k}$-гладкую разрешимость соответствующего гомологического уравнения.

\section{5. Траекторная и точная классификации бездивергентных векторных по- лей на торе (с дырками).}

\section{1. Сепаратрисная диаграмма.}

ЛЕмма 2. Если на торе $T^{2}$ имеется периодическая траектория бездивергентного векторного поля $v$, реализующая нетривиальный иикл в гомологиях, то поле $v$ является гамильтоновым, $v=\operatorname{sgrad}_{\omega} H, H: T^{2} \rightarrow S^{1}$. 
Назовем объединение гиперболических особых точек бездивергентного поля и входящих-вьходящих сепаратрис сепаратрисной диаграммой. Сепаратрису назьваем конечной, если ее $\omega$ - и $\alpha$-предельньпи множествами являются только особые точки. Сепаратрису назьваем $\omega$-блуждающей, если она выходит из особой точки, но не входит ни в

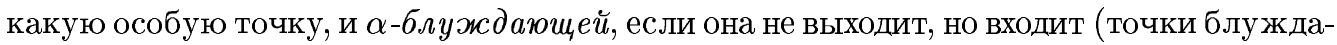
ющих сепаратрис являются неблуждающими в смысле [11]). Сепаратрисным ииклом назьваем объединение сепаратрис, образующих контур, гомеоморфный окружности. Рассмотрим граничные компоненты (дырки) и эллиптические особые точки (центры).

Лемма 3. Для связного компактного двумерного многообразия, отличного от $D^{2}, S^{2}=D^{2} \cup D^{2}, S^{1} \times I, \mathscr{M}=S^{1} \widetilde{\times} I$ u $\mathbb{R} P^{2}=D^{2} \cup \mathscr{M}$, любой чентр и любая дырка лехст внутри сепаратрисного иикла.

ЛЕмма 4. Любая компонента связности сепаратрисной диаграммы на $T^{2}$ либо замкнута, либо содержит ровно одну $\omega$-блуждающую сепаратрису, ровно одну

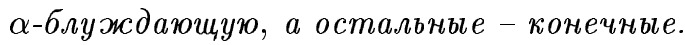

ДокАЗАТЕльСтво. Если бездивергентное векторное поле - гамильтоново, то все компоненты связности замкнуты. Иначе, рассмотрим компоненту связности и выбросим из нее все блуждающие сепаратрисы. Оставшееся замкнутое множество лежит внутри контура, ограничивающего диск (лемма 2). При обходе полученного контура входящие и выходящие (т.е. $\alpha$ - и $\omega$-блуждающие) сепаратрисы будут чередоваться. Заметим, что в силу блуждания и бездивергентности незамкнутая компонента сепаратрисной диаграммы не может содержаться ни в каком сепаратрисном контуре, но может содержать внутри себя замкнутую компоненту.

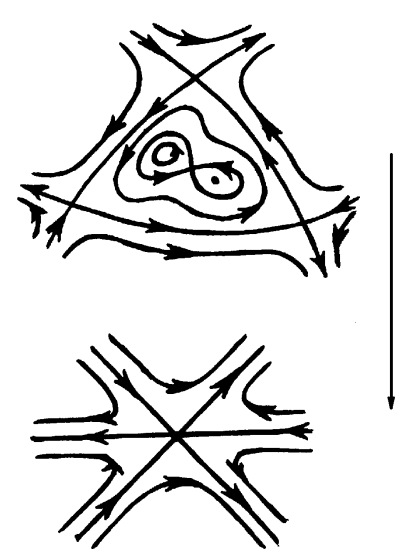

Рис. 3

Применим для каждого незамкнутого контура процедуру редукиии, устранения конечной части сепаратрисной диаграммы со всем, что лежит внутри нее (рис. 3 ). В результате редукции пропадут все замкнутые компоненты и контуры, а следовательно, в силу леммы 3 все центры и дырки. Сепаратрисные компоненты с двумя блуждающими сепаратрисами дадут после редукции и сглаживания регулярную точку векторного поля, с четырьмя - седло, с шестью и более - вырожденное седло. Поскольку редуцированное поле не будет иметь особых точек неотрицательных индексов, а $\chi\left(T^{2}\right)=0$, то особых точек нет. 
5.2. Траекторная классификация. Применяя процедуру редукции, можно доказать (cp. c [1])

ПРЕДЛОЖЕНИЕ 3. Для невырожденного негамильтонова бездивергентного векторного поля на торе $T^{2}$ существует замкнутая трансверсаль потока, реализующая базисный иикл. Она пересекает только блуждающие сепаратрисы.

Рассмотрим построенную трансверсаль как базисный цикл $\gamma_{1}$. Выпустим из некоторой ее точки блуждающую траекторию, не являющуюся сепаратрисой. Разрежем тор вдоль отрезка этой траектории до первого пересечения с $\gamma_{1}$ и вдоль цикла $\gamma_{1}$. Получим фундаментальный квадрат, две стороны которого являются частями траектории потока, а две другие - трансверсалями. Пусть поток течет снизу вверх около нижней стороны. В силу выбора $\gamma_{1}$ конечные части сепаратрисной диаграммы не пересекают сторон квадрата.

ПРЕДЛОЖЕНИЕ 4. Траекторный портрет негамильтонова бездивергентного поля имеет описанный вид на фундаментальном квадрате, причем и конечные части сепаратрисной диаграммы, и дырки лехсат во внутренности квадрата, входящая $\alpha$-блуждающая сепаратриса приходит с нижней стороны, а исходящая $\omega$-блуждающая уходит на верхнюю. Боковые стороны имеют иррациональные коэффичиенты наклона, а склейка верхней и нижней сторон происходит при помощи вертикального сдвига $(x, 1) \mapsto(x, 0)$.

Итак, любая компонента сепаратрисной диаграммы либо замкнута и тогда является плоской буквой-атомом (п. 1), либо незамкнута с двумя концами и тогда представляет собой плоскую букву-атом, у которой одна из внешних сепаратрис разорвана. Замкнутые компоненты сепаратрисной диаграммы должны лежать внутри незамкнутых и могут, в свою очередь, содержать внутри себя другие замкнутые компоненты, но не могут содержать незамкнутые.

ОПРЕДЕЛЕНИЕ. Назовем 1-меченной буквой-атомом букву-атом с указанием разрьваемого ребра. Для плоских букв-атомов такие меченные ребра должны быть внешними. Плоские 1-меченные буквы-атомы с указанием структуры вложений конечных компонент (букв-атомов) будем назьвать плоскими пополненными буквами-атомами.

Аналогично, отмечая $k$ ребер на букве-атоме, мы получим $k$-меченную букву-атом. Соответствующие сепаратрисные диаграммы возникают в случае эйлеровой характеристики $\chi \leqslant 2-2 k$.

Рассмотрим на трансверсали $\gamma_{1}$ параметр $x(\bmod 1)$ такой, что отображение последования имеет вид $x \mapsto x+\theta, \theta=1 / \lambda$, где $\lambda \in \mathbb{R} \backslash \mathbb{Q}$ - число врашения редуцированной системы. Отображение последования не определено в первых точках пересечения цикла $\gamma_{1} \alpha$-блуждающими сепаратрисами, но этим точкам мы сопоставляем первые точки пересечения цикла $\gamma_{1}$ соответствующими $\omega$-блуждающими сепаратрисами, и это сопоставление описьвается тем же правилом $x \mapsto x+\theta$. Отметим первые точки пересечения цикла $\gamma_{1} \alpha$-блуждающими сепаратрисами. Сопоставим им соответствующие плоские пополненные буквы-атомы. Назовем окружность полученного типа оснащенной.

ТЕОРема 5. Траекторный портрет гамильтоновой системы на торе ( ками) классифицируется графом-молекулой Г (см. п. 1). Траекторный портрет 
негамильтонова бездивергентного поля классифицируется числом вращения $\lambda u$ оснащенной окружностью.

При замене трансверсали $\gamma_{1}$ на другую трансверсаль, удовлетворяющую условиям предложения 3 , индуцированное оснащение может измениться. Точнее, список пополненных букв-атомов останется прежним, но соответствующие им точки окружности могут поменять порядок. На рис. 4 изображен пример, когда при смене трансверсали в том же гомологическом классе указанный порядок меняется.

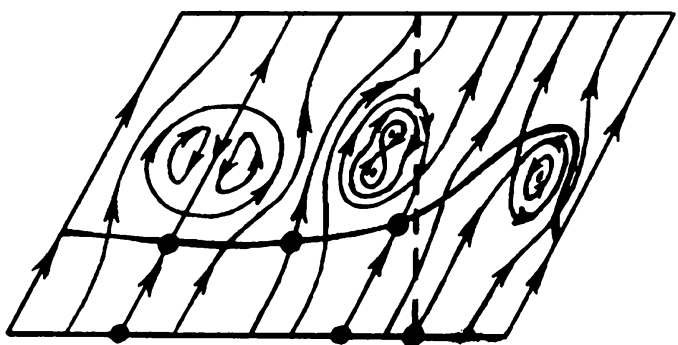

Рис. 4

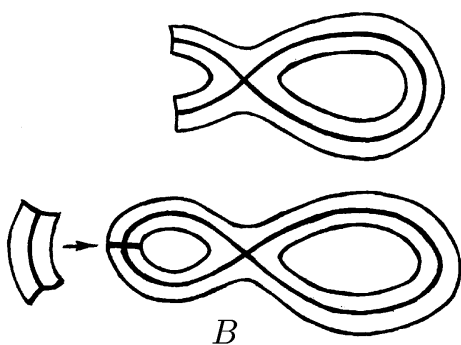

Рис. 5

5.3. Точная классификация ростков гамильтоновых векторных полей на 1-меченных буквах-атомах. Рассмотрим букву-атом $\left(P^{2}, K\right)$, у которой одно ребро (сепаратриса) разорвано. Рассмотрим на ней симплектическую форму $\omega$ и функцию Морса $F$ такую, что $K=F^{-1}(0)$ и 0 - невырожденное критическое значение. Граф $K$ является сепаратрисной диаграммой потока $v=\operatorname{sgrad}_{\omega} F$, и, выбрасывая разорванное ребро, мы получаем конечную часть сепаратрисной диаграммы. Рассмотрим классификацию с точностью до сопряжения ростков гамильтоновых векторных полей на конечной части $K^{\prime} \subset K$.

Чтобы определить инварианты, склеим разорванное ребро е. Это всегда можно сделать, так как в окрестности неособой точки все гамильтоновы поля сопряжены. Две полученных таким образом буквы-атома получаются друг из друга применением операции вклейки в ребро е и (или) обратной - вырезания, где операция вклейки определяется следующим образом (ср. с [5]). Рассматривается криволинейная трапеция $\left\{(t, F) \mid f_{1}(F) \leqslant t \leqslant f_{2}(F),-\varepsilon \leqslant F \leqslant \varepsilon\right\}$ и произвольньй разрез ребра $e$ и отождествляются стороны разреза с соответствуюшими сторонами $\left\{(t, F) \mid t=f_{i}(F)\right\}$ криволинейной трапеции так, чтобы функции $F$ и векторные поля $v=\operatorname{sgrad}_{\omega} F$ склеивались гладко (рис. 5).

Инварианты $\Lambda_{A, k}$ не меняются при указанной операции. Определим инвариант $Z_{k}$. Сделаем это при помощи функции $\varkappa$, как и в п. 2.1. Для всех колец из $P^{2} \backslash K$, кроме двух инцидентных отмеченному ребру $е$, функция $\varkappa$ определяется по формуле (2). Пусть $A_{0}-$

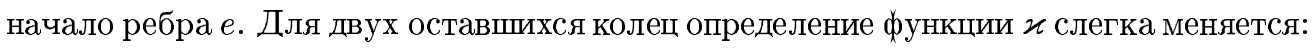

$$
\varkappa\left(x_{0}\right)=\lim _{\substack{x \rightarrow x_{0} \\ x \in U_{s i}}} \varkappa(x)+\Lambda_{A_{i_{s}}, k}\left(F\left(x_{0}\right)\right) \ln F\left(x_{0}\right)-\delta_{i_{s}}^{0} c_{i}^{k}\left(F\left(x_{0}\right)\right),
$$

где $s$ нумерует $U_{s i}$ - промежутки между сечениями на кольце $C_{i}$ - одном из двух отмеченных, а $\delta_{i_{s}}^{0}=0$, если вершина $A_{i_{s}}$ отлична от $A_{0}$, и $\delta_{i_{s}}^{0}=1$, если они совпадают. Дальнейшее определение $Z_{k}$ остается прежним. При операции вклейки-вырезания полученньй элемент $Z_{k} \in H_{1}\left(\widetilde{P}^{2} ; \mathbb{R}_{k}[t]\right)$ не изменяется. 
В п. 2.1 был определен также инвариант $\Delta_{k} \in B^{2}\left(\widetilde{P}^{2} ; \mathbb{R}_{k}[t]\right)$, которьй на диске, заклеивающем кольцо $C_{i}$ (обозначим его также через $C_{i}$ ), принимает значение, равное отрезку ряда Тейлора до $k$-го члена в нуле от функции $c_{i}^{k}(F)$ из $(1)$. Ясно, что для всех колец $C_{i}$ кроме выделенных двух $C_{i_{e}^{+}}($где $F>0)$ и $C_{i_{e}^{-}}($где $F<0)$ операция вклейки-вырезания не меняет полинома (ряда) $\Delta_{k}\left(C_{i}\right)$. А для $i=i_{e}^{ \pm}$изменение выражается формулой $\Delta_{k}\left(C_{i}^{ \pm}\right) \mapsto \Delta_{k}\left(C_{i}^{ \pm}\right)+p$, где $p$ - полином (ряд), соответствуюший вклеиваемой криволинейной трапеции. Определим инвариант $\Delta_{k}$ как отображение, сопоставляющее $C_{i}$ полином (ряд) $\Delta_{k}\left(C_{i}\right)$ при $i \neq i_{e}^{ \pm}$и сопоставляющее паре $\left(C_{i_{e}^{+}}, C_{i_{e}^{-}}\right)$ выражение

$$
\left[\Delta_{k}\left(C_{i_{e}^{+}}\right)-\Delta_{k}\left(C_{i_{e}^{-}}\right)\right]=\sum_{i^{-} \neq i_{e}^{-}} \Delta_{k}\left(C_{i^{-}}\right)-\sum_{i^{+} \neq i_{e}^{+}} \Delta_{k}\left(C_{i^{+}}\right)
$$

(напомним, что $\Delta_{k} \in B^{2}$, см. п. 2.1). Таким образом, инвариант $\Delta_{k}$ сопоставляет полиномы (ряды) только неразрезанньп кольцам $C_{i}$ и не меняется при операции вклейки-вырезания.

Для фиксированного кольца $C_{i} \neq C_{i_{e}^{ \pm}}$можно считать, что $c_{i}^{k}(F) \equiv 0$ (предложение 1). Определим инварианты $\Lambda_{A, k}, Z_{k}, \Delta_{k}$ относительно полученной функции Морса $F$, параметризуюшей слоение ростка $K^{\prime} \subset K$ траекториями поля $v$. Итак, мы нашли набор $\operatorname{Inv}_{k}\left(P^{2}, K^{\prime} \subset K, v\right)_{i}=\left\{\Lambda_{*, k}, Z_{k}, \Delta_{k}\right\}$, инвариантный относительно операции вклейки-вырезания, примененной к ребру $e$, и потому являющийся инвариантом ростка гамильтонова поля $v$ на конечной части $K^{\prime} \subset K$.

ТЕорема 6. Ростки гамильтоновых векторных полей $v$ на конечной части $K^{\prime}$ графа $K \subset P^{2}$ с одним разорванным ребром полностью классифицируются с точностью до $C^{k}$-сопряжсения набором инвариантов $\operatorname{Inv}_{k}\left(P^{2}, K^{\prime} \subset K, v\right)_{i}$.

5.4. Точная классификация бездивергентных полей на торе. Введем "среднюю длину" векторного поля $v$ на торе $T^{2}$ (с дырками), как это сделано в п. 4. Для этого заклеим дырки дисками, продолжим на них произвольным образом пару $(v, \omega)$ и положим

$$
v_{0}=\|S\|, \quad S=\frac{1}{|\omega|}\left[i_{v} \omega\right] \in H^{1}\left(T^{2} ; \mathbb{R}\right),
$$

где $|\omega|$ - интеграл $\omega$ по тору с дырками, а норма $\|\cdot\|$ в пространстве $H^{1}$ индуцируется нормой в $H_{1}$ с ортонормированным базисом из (любых) циклов.

ПРЕДЛОЖЕНИЕ 5. Для негамильтонова бездивергентного векторного поля $v$ средняя длина $v_{0} \neq 0$. Для почти всех чисел вращения $\lambda$ она мохсет быть определена также следующим образом. Пусть $и(t)$ - регулярная блуждающая траектория, т.е. незамкнутая траектория, отличная от сепаратрись, и пусть $u_{0}^{t}=\{u(s), s \in[0, t]\}$. Пусть $\left(\gamma_{1}, \gamma_{2}\right)$ - базис из трансверсалей (предложсене 3$), u$ число вращения в этом базисе задается формулой $\lambda=\operatorname{tg} \alpha$. Пусть $\tau_{i}(t)-$ число пересечений $u_{0}^{t}$ с $\gamma_{i}$. Тогда

$$
v_{0}=\varrho \lim _{t \rightarrow \infty} \frac{\tau_{1}(t)}{t \sin \alpha}=\varrho \lim _{t \rightarrow \infty} \frac{\tau_{2}(t)}{t \cos \alpha},
$$

әде $\varrho$ - относительный обгем "области блуждания", т.е. $\varrho=|\widetilde{\omega}| /|\omega|$, әде $\widetilde{\omega}=0$ для всех точек периодических траекторий и $\widetilde{\omega}=\omega$ в остальных точках. Инвариант $S$ связан с $v_{0}$ формулой $\left.\left.n .4: S=v_{0} \cos \alpha \widehat{\left[\gamma_{2}\right.}\right]-v_{0} \sin \alpha \widehat{\left[\gamma_{1}\right.}\right] \in H^{1}\left(T^{2} ; \mathbb{R}\right)$. 
ДокАЗАТЕЛЬСТВо. Если $v_{0}=0$, то $\nu=i_{v} \omega=d H$, т.е. почти все траектории поля $v=\operatorname{sgrad}_{\omega} H$ замкнуты. Рассмотрим конечные части сепаратрисной диаграммы. Выделим в них максимальные сепаратрисные контуры. Эти контуры ограничивают диски $D_{j}^{2}$. Рассмотрим вложение $i: T^{2} \backslash \bigsqcup D_{j}^{2} \rightarrow T^{2}$. Для индуцированного отображения в $H^{1}$ и класса $a=[\nu]=[i v \omega]$ имеем $i^{*} a \neq 0$. На дисках $D_{j}^{2}$ имеется определенньй с точностью до постоянной гамильтониан, и, вычитая его дифференциал, мы можем считать $\left.\nu\right|_{D_{j}^{2}}=0$. Применим процедуру редукции (лемма 4) $T^{2} \rightarrow T^{2} / \bigsqcup D_{j}^{2}$. Можно аппроксимировать редуцированное поле полем без особенностей с тем же замыканием траекторий, откуда

$\frac{1}{|\widetilde{\omega}|}\left[i_{v} \omega\right]=\frac{1}{|\widetilde{\omega}|}[i, \widetilde{\omega}]=\widetilde{S}=\widetilde{v}_{0} \cos \alpha\left[\widehat{\gamma_{2}}\right]-\widetilde{v}_{0} \sin \alpha\left[\widehat{\gamma_{1}}\right], \quad \widetilde{v}_{0}:=\lim _{t \rightarrow \infty} \frac{\tau_{1}(t)}{t \sin \alpha}=\lim _{t \rightarrow \infty} \frac{\tau_{2}(t)}{t \cos \alpha}$.

Здесь символ $[i, \widetilde{\omega}]$ обозначает предел

$$
\lim _{\varepsilon \rightarrow 0}\left[i_{v_{\varepsilon}} \widetilde{\omega}_{\varepsilon}\right]
$$

где $v_{\varepsilon}$ - регулярная гладкая аппроксимация редуцированного поля $v$, имеющая те же замькания траекторий, $\widetilde{\omega}_{\varepsilon}-$ гладкая аппроксимация редуцированной формы объема $\widetilde{\omega}$ такая, что поле $v_{\varepsilon}$ бездивергентное относительно $\widetilde{\omega}_{\varepsilon}$, и $\varepsilon=\max \left(\varepsilon_{1}, \varepsilon_{2}\right)$, где $\varepsilon_{1}-$ модуль апроксимации, а $\varepsilon_{2}$ - мера (относительно $\widetilde{\omega}$ ) ееносителя. Те же соглашения применимы и для выражений

$$
\lim _{t \rightarrow \infty} \frac{\tau_{1}(t)}{t \sin \alpha}, \quad \lim _{t \rightarrow \infty} \frac{\tau_{2}(t)}{t \cos \alpha},
$$

причем для почти всех чисел вращения $\lambda$ их предельные значения при $\varepsilon \rightarrow 0$ совпадают с пределами, посчитанными для регулярной траектории $u(t)$, как указано в формулировке предложения. Более точно, это верно для всех $\lambda$, для которьх число $1 / \lambda$ имеет тип $(K, \sigma), K, \sigma>0$. Покажем это.

Предположим для простоты, что имеется ровно одна точка $x_{0}$ - образ редукции конечной компоненты сепаратрисной диаграммы. Для поля $v$ время пробегания около $x_{0}$ стремится к бесконечности как логарифм расстояния от $x_{0}$ до траектории (расстояние измеряется вдоль трансверсали с каноническим параметром $x$ ). Для аппроксимированного поля это время ограничено. Рассмотрим длинньй отрезок $\widetilde{u}_{0}^{t}(\varepsilon)$ траектории аппроксимированного поля $\widetilde{u}_{\varepsilon}(s)$. Этот отрезок будет проходить через левую $\varepsilon$-окрестность точки $x_{0}$ порядка $n=[t \varepsilon]$ раз. Если $1 / \lambda$ - число типа $(K, \sigma)$, то этот отрезок подойдет к точке $x_{0}$ не ближе, чем на расстояние $C_{0} K / n^{1+\sigma}$. Поэтому разность между величинами $\tau_{1}(t) /(t \sin \alpha)$, посчитанными для полей $v$ и $v_{\varepsilon}$, имеет порядок

$$
\frac{\varepsilon}{n} \sum_{i=0}^{n-1} \Lambda^{-}\left(\frac{C_{0} K}{n^{1+\sigma}}+i \frac{\varepsilon}{n}\right) \ln \left(\frac{C_{0} K}{n^{1+\sigma}}+i \frac{\varepsilon}{n}\right)
$$

где $\Lambda^{-}$- функция, соответствующая бесконечной части функции времени пробегания слева мимо конечной компоненты сепаратрисной диаграммы (1). Эта величина аппроксимируется несобственным интегралом (и сходится к нему при $n \rightarrow \infty$ )

$$
\int_{0}^{\varepsilon} \Lambda^{-}(x) \ln x d x=\Lambda_{0}^{-} \int_{0}^{\varepsilon} \ln x d x+o(\varepsilon)=\Lambda_{0}^{-} \varepsilon \ln \frac{\varepsilon}{e}+o(\varepsilon) \rightarrow 0 \quad \text { при } \varepsilon \rightarrow 0 .
$$


Аналогичное заключение справедливо и для правой $\varepsilon$-окрестности $x_{0}$. Поэтому величину

$$
\lim _{t \rightarrow \infty} \frac{\tau_{1}(t)}{t \sin \alpha}
$$

можно вычислять по аппроксимирующему полю $v_{\varepsilon}$ с теми же траекториями. Итак,

$$
S=\frac{|\widetilde{\omega}|}{|\omega|} \widetilde{S}, \quad\|\widetilde{S}\|=\widetilde{v}_{0},
$$

и предложение доказано.

Добавим к отмеченным точкам оснащения трансверсали из теоремы 5 не только соответствующую пополненную букву-атом, но и ее инвариант $\operatorname{Inv}_{k}\left(P^{2}, K^{\prime} \subset K, v\right)_{*}$ из теорем 2,6 . Назовем полученное оснащение $k$-оснащением, $k=1, \ldots, \infty$. Поскольку для плоских 1-меченных атомов групшы гомологий $H_{1}\left(\widetilde{P}^{2}\right)=0$, для данного оснащения $Z_{k}=0$. Более того, если бездивергентное поле $v$ - общего положения, то 1-меченные буквы-атомы - только разорванные атомы $B$. В этом случае $k$-оснащение получается из оснашения добавлением инвариантов $\Lambda_{k}$ для всех вершин всех атомов, выраженных через соответствуюший параметр $F$, как в предложении 1.

ТЕОрема 7. Точная классификачия гамильтоновых систем на торе $T^{2}$ ( с дырками) дается теоремой 3. Для почти всех иррачиональных чисел вращения $\lambda$ негамильтоново бездивергентное векторное поле на торе $T^{2}$ ( с дырками) классифицируется с точностью до $C^{\infty}$-сопряжения инвариантом $S$ и трансверсалью. Если у двух систем с числами $\theta=1 / \lambda$ типа $(K, \sigma)$ совпадают $S$-инварианты $u(k+r)$-оснащения, әде $r=3+[\sigma]$, то системы $C^{k}$-сопряэсены $(k \geqslant 1)$. При замене трансверсали $(k+r)$-оснащение отмеченных точек остается то же, но их порядок на окружности может измениться.

ДокАЗАТЕЛЬСтво. Совместим траекторные портреты двух систем так, чтобы порядки оснащенных точек на трансверсалях совпадали. По теореме 6 в окрестности максимальных дисков, ограниченных максимальными конечньми сепаратрисньпи контурами, существует сопрягающий системы автоморфизм. Его можно выбрать так, чтобы локальные трансверсали к $\alpha$-блуждающим сепаратрисам в окрестности конечной части $K^{\prime} \subset K$ сопрягались. Рассмотрим теперь для выбранных трансверсалей функцию времени последования: $w_{1}(x)$ для первой системы и $w_{2}(x)$ для второй. Для отмеченных точек $w_{i}(x)=\infty$. Из совпадения $(k+r)$-оснащений следует, что $g(x)=w_{1}(x)-w_{2}(x) \in$ $C^{k+r}$. Более того, среднее этой функции равно 0. Следовательно, коэффищиенты Фурье этой функции удовлетворяют $\left|g_{n}\right|<\varepsilon / n^{k+r}, n>n_{0}$. Поэтому решение гомологического уравнения имеет коэффициенты Фурье, удовлетворяющие соотношению

$$
\left|f_{n}\right|=\frac{\left|g_{n}\right|}{\left|e^{i n \theta}-1\right|}<C \varepsilon n^{1+\sigma-k-r}=\frac{C \varepsilon}{n^{k+1+(1+[\sigma]-\sigma)}}
$$

откуда по принципу Вейерштрасса получаем, что решение $f \in C^{k}\left(S^{1}\right)$. Можно считать, что новая трансверсаль не проходит через образы максимальных дисков при редукции. Системы совмешаются сдвигом вдоль траекторий. Гладкость этого преобразования следует из совпадения инвариантов. 


\section{6. Связная сумма трех проективных плоскостей.}

6.1. Траекторная классификация. Пусть $P^{2}=\#{ }_{1}^{3} \mathbb{R} P^{2}=K^{2} \# \mathbb{R} P^{2}=T^{2} \# \mathbb{R} P^{2}-$ сфера с тремя листами Мёбиуса. Можно считать, что все дырки на $P^{2}$ заклеены. Мы будем использовать представление $P^{2}$ в виде тора с вклеенньм листом Мёбиуса. Пусть $v$ - бездивергентное векторное поле на $P^{2}$. После редукции и сглаживания (лемма 4 ) мы получим поле $v$ с ровно одной (гиперболической) особой точкой: $\chi\left(P^{2}\right)=-1$.

Если существует периодическая траектория, реализующая нетривиальный цикл в гомологиях $H_{1}\left(P^{2} ; \mathbb{R}\right) \simeq \mathbb{R}^{2}$, то почти все траектории замкнуты. Действительно, разрезая вдоль этой траектории, мы получим бездивергентное поле на кольце с вклеенным листом Мёбиуса. Он может быть вклеен во внутренность кольца, а может быть подклеен "крест-накрест" (рис. 6). В любом случае почти все траектории замкнуты. Будем считать, что периодические траектории гомологичны нулю в $H_{1}\left(P^{2} ; \mathbb{R}\right)$. Возможны два случая.

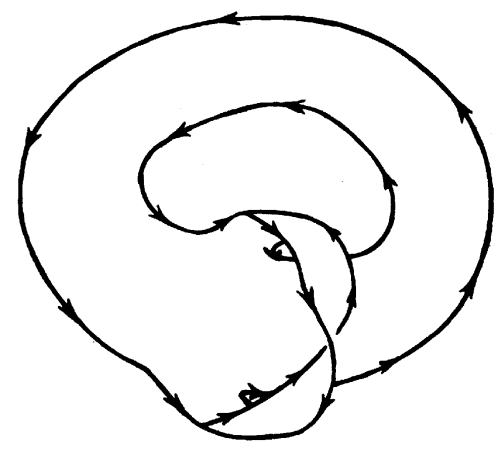

Рис. 6
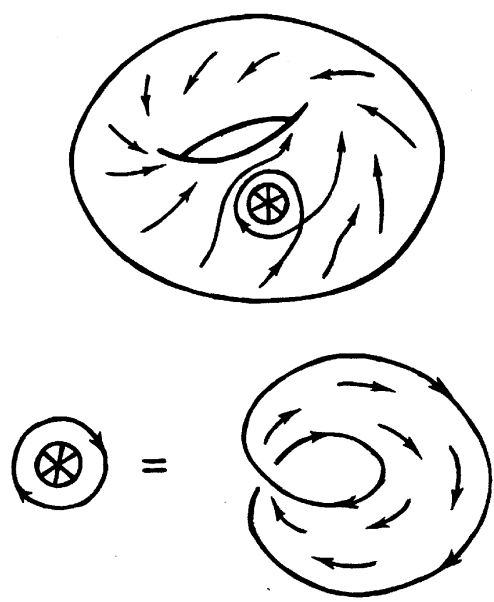

Рис. 7

СлучАЙ 1. Редуцированная система не имеет периодических траекторий, ограничивающих диск. Поэтому гомологичная нулю замкнутая траектория ограничивает лист Мёбиуса. Если система имеет периодическую траекторию, не гомологичную нулю в $H_{1}\left(P^{2} ; \mathbb{Z}\right) \simeq \mathbb{Z}^{2} \oplus \mathbb{Z}_{2}$, то, рассматривая малую регулярную окрестность этой траектории, мы заключаем, что система обладает гомологичной нулю в $H_{1}\left(P^{2} ; \mathbb{Z}\right)$ траекторией, ограничивающей лист Мёбиуса. Обратно, гомологичная нулю в $H_{1}\left(P^{2} ; \mathbb{Z}\right)$ траектория ограничивает лист Мёбиуса, на котором почти все траектории замкнуты. Для редуцированной системы это обозначает существование периодической траектории, реализующей элемент порядка 2 в $H_{1}\left(P^{2} ; \mathbb{Z}\right)$. Расширяя множество периодических траекторий, мы получаем максимальньй лист Мёбиуса, ограниченньй сепаратрисной связкой (рис. 7). Проведем дополнительную редукцию, уничтожающую максимальньй лист Мёбиуса. После сглаживания получим бездивергентное поле на торе без особенностей. Припишем такому векторному полю число вращения $\lambda$ в выбранном базисе на торе $\left(\gamma_{1}, \gamma_{2}\right)$. Аналогично п. 5.2, снабдим трансверсальньй базисный цикл $\gamma_{1}$ для нередуцированной системы схемой строения 1-меченных букв-атомов и структурой вложений замкнутых сепаратрисных компонент, центров и граничных окружностей для каж- 
дой первой точки пересечения $\gamma_{1} \alpha$-блуждающими сепаратрисами. Одна из точек пересечения будет отвечать сепаратрисному контуру, ограничивающему лист Мёбиуса (единственной $\alpha$-блуждающей компоненте, остающейся после первой редукции). Соответствующие ей буквы-атомы могут быть неориентируемы.

СлучАй 2. Предположим теперь, что редуцированная система не имеет периодических траекторий, но имеет сепаратрисную связку. Тогда эта сепаратрисная связка реализует центральную окружность листа Мёбиуса - цикл конечного порядка в $H_{1}\left(P^{2} ; \mathbb{Z}\right)$. В этом случае сепаратрисная диаграмма представляет из себя букву $B$, у которой одно ребро перекручено, как лист Мёбиуса, а второе - разорвано (рис. 8). Сжимая в точку конечную сепаратрису и сглаживая, мы получаем бездивергентное векторное поле на торе без особых точек; сопоставим ему число вращения $\lambda$ в базисе $\left(\gamma_{1}, \gamma_{2}\right)$. Определим, как и ранее, траекторное оснашение трансверсали $\gamma_{1}$.
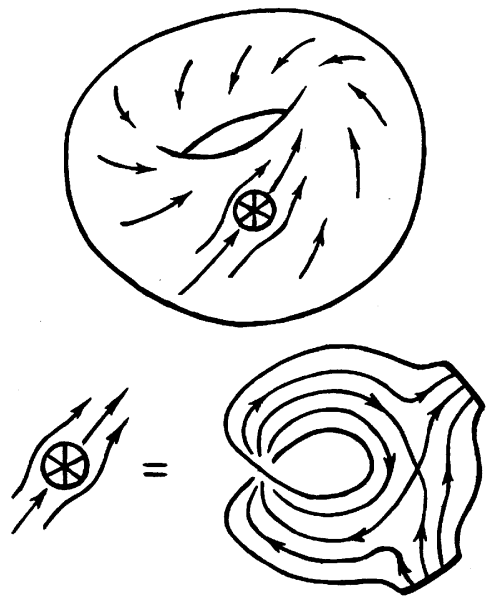

Рис. 8
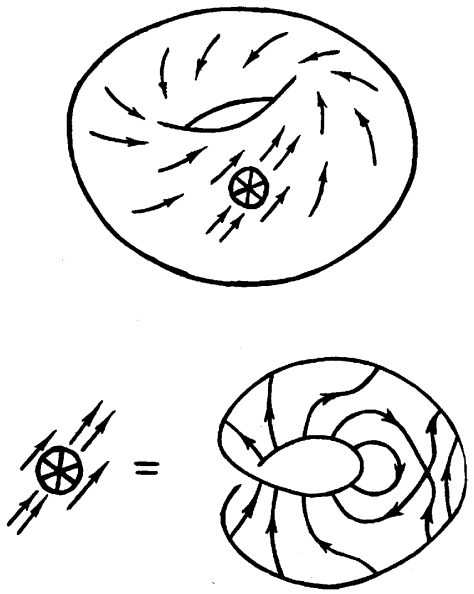

РИС. 9

ТЕОРема 8. Если почти все траектории бездивергентного векторного поля $v$ на $P^{2}$ (с дырками) замкнуты, то такая система классифицируется графом-молекулой Г (см. п. 1). Иначе возможны случай 1 - наличие и блуждающих, и периодических траекторий, и случай 2 - отсутствие периодических траекторий, но существование нетривиальной в $H_{1}\left(P^{2} ; \mathbb{Z}\right)$ сепаратрисной связки. $B$ каждом из этих случаев полным траекторным инвариантом является число вращения $и$ траекторно оснащенная трансверсаль.

ДокАЗАТЕЛЬСТво. Пусть у рассматриваемой системы нет ни замкнутых траекторий, ни сепаратрисньх связок. Можно считать, что единственная седловая точка лежит внутри листа Мёбиуса. Индекс векторного поля $v$ вдоль граничной окружности листа Мёбиуса равен \pm 1 . Продеформируем эту окружность так, чтобы поле вращалось вокруг нее "монотонно". Тогда внутри листа траектории редуцированного поля $v$ выглядят, как показано на рис. 9. Редуцируя лист Мёбиуса $P^{2} \rightarrow T^{2}$, находим трансверсали потока $\left(\gamma_{1}, \gamma_{2}\right)$, реализующие базисные циклы в $H_{1}\left(T^{2} ; \mathbb{R}\right)=H_{1}\left(P^{2} ; \mathbb{R}\right)$ и не проходящие через лист Мёбиуса. На первой трансверсали имеется естественный параметр $x(\bmod 1)$. Рассмотрим первые точки пересечения цикла $\gamma_{1} \alpha$-блуждающими се- 
паратрисами, входящими в седло. Рассмотрим отрезок между этими точками на окружности $\gamma_{1}$, для каждой точки которого выходящая траектория проходит через лист Мёбиуса до следующего пересечения с $\gamma_{1}$. Пусть $[0, q]$ - этот отрезок длины $q$. Тогда отображение последования задается формулой

$$
\Phi(x)= \begin{cases}(q-x)+\theta(\bmod 1), & \text { если } x \in(0, q), \\ x+\theta(\bmod 1), & \text { если } x \in(q, 1),\end{cases}
$$

где $\theta=1 / \lambda(\bmod 1), \lambda$ - число вращения. Доопределим $\Phi$ по непрерьвности на $[0, q]$. Легко видеть, что отображение $\Phi: S^{1} \rightarrow S^{1}$ периодично. Противоречие.

Периодичность $\Phi$ объясняется геометрически. Действительно, сепаратрисная диаграмма замкнута и представляет из себя букву $B$ (рис. 5), к каждому из трех концов которой приклеен лист Мёбиуса со стандартным расслоением на окружности. Трансверсаль $\gamma_{1}$ пересекает конечные сепаратрисы $2 m_{1}$ и $2 m_{2}$ раз. Поэтому периоды отображения последования на (продолженньх) трансверсалях равны $2 m_{i}$ для всех точек трансверсали на внутренних листах Мёбиуса кроме одной, где период равен $m_{i}, i=1,2$. Для внешнего листа Мёбиуса этапара периодов равна $\Pi=\left(2\left(m_{1}+m_{2}\right), m_{1}+m_{2}\right)$. Итак, доказано

ПРЕДЛОЖЕНИЕ 6. Окружность $S^{1}$ разбивается в дизбюнктное оббединение $m_{1}$ замкнутых интервалов таких, что $\Phi$ имеет на них периоды $\Pi_{1}=\left(2 m_{1}, m_{1}\right)$, $m_{2}$ замкнутых интервалов с периодами $\Pi_{2}=\left(2 m_{2}, m_{2}\right)$ u $m_{1}+m_{2}$ открытых интервалов с периодами $\Pi_{3}=\left(2\left(m_{1}+m_{2}\right), m_{1}+m_{2}\right)$.

6.2. Точная классификация ростков бездивергентных полей на неориентируемых 1-меченных буквах-атомах. Склеим разорванное ребро е так, чтобы из двух граничных отрезков получилось две окружности, а не одна. Инварианты $\Delta_{k}, Z_{k}$ и $\Lambda_{*, k}$ определяем аналогично п. 5.3. Если имеется отличное от $C_{i} \pm$ кольцо, то, как и в предложении 2 , мы можем зафиксировать струю гамильтониана - функции Морса $\pm F$ на особом слое буквы-атома, потребовав равенства нулю конечной части функции периодов - значения $\Delta_{k}$ на этом кольце. Рассмотрим случай, когда нет других колец кроме $C_{i}^{ \pm}$, как например, в случае буквы-атома $B$, у которого одно ребро перекручено, как лист Мёбиуса, а другое разорвано. Тогда если $\Lambda$-инварианты, соответствующие кольцам $C_{i_{e}^{+}}$ и $C_{i_{e}^{-}}$, различны, то функция $\pm F$ выбирается из условия $\Delta_{k}\left(C_{i_{e}^{+}}\right)-\Delta_{k}\left(C_{i_{e}^{-}}\right)=0$, и $\Delta_{k}$-инварианта здесь нет. Если же $\Lambda_{k}\left(C_{i_{e}^{+}}\right)=\Lambda_{k}\left(C_{i_{e}^{-}}\right)$, то возможны два случая: $\Delta_{k}\left(C_{i_{e}^{+}}\right)=\Delta_{k}\left(C_{i_{e}^{-}}\right)$и $\Delta_{k}\left(C_{i_{e}^{+}}\right) \neq \Delta_{k}\left(C_{i_{e}^{-}}\right)$. В случае равенства выбираем $\pm F$ из условия $\Delta_{k}\left(C_{i_{e}^{+}}\right)=\Delta_{k}\left(C_{i_{e}^{-}}\right)=0$, и $\Delta_{k}$-инварианта опять нет. В случае неравенства при помощи вклейки-вырезания $\Delta_{k} \mapsto \Delta_{k}+p$ можно получить $\Delta_{k}\left(C_{i_{e}^{+}}\right)=-\Delta_{k}\left(C_{i_{e}^{-}}\right)$. Выбирая другую функцию Морса $\widehat{\widetilde{F}}=\widehat{\widetilde{F}}(\widehat{F})$ (см. обозначения в начале п. 2.2), можно считать $\Delta_{k}\left(C_{i_{e}^{-}}\right)=0$. В этом случае $\Delta_{k}$-инвариант есть, и им является полином (ряд) $\Delta_{k}\left(C_{i_{e}^{+}}\right)$, выраженный через новую функцию Морса $\pm \widetilde{F}$. Теорема 6 переносится на случай неориентируемьх 1-меченных букв-атомов.

6.3. Точная классификация. Аналогично п. 5.4 вводим среднюю длину поля $v$ на $P^{2}$ (с дырками) формулой (3) или при помощи дополнительной редукции $P^{2} \rightarrow T^{2}$ в случаях 1 и 2 , а также вводим инвариант $S \in H^{1}\left(P^{2} ; \mathbb{R}\right)$ и $k$-оснащенную трансверсаль. 
ТЕОРемА 9. Если почти все траектории бездивергентного векторного поля $v$ на $P^{2}$ (с дырками) замкнуты, то точная $C^{k}$-классификация дается теоремой 3.

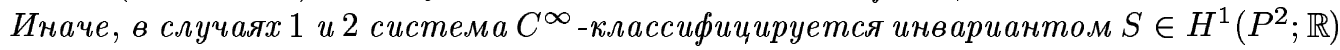
$u \infty$-оснащенной трансверсалью. Для чисел вращения $\lambda$ типа $(K, \sigma)$ из совпадения у двух систем $S$-инвариантов и $(k+r)$-оснащений следует их $C^{k}$-сопряженность, где $r=3+[\sigma]$.

Автор благодарен академику РАН профессору А. Т. Фоменко и д. ф.-м. н А. В. Болсинову за внимание к работе.

\section{СПИСОК ЦИТИРОВАННОЙ ЛИТЕРАТУРЫ}

[1] Арнольд В.И. Топологические и эргодические свойства замкнутых 1-форм с рационально независимыми периодами // Функцион. анализ и его прилож. 1991. Т. 25. № 2. С. 1-12.

[2] Новиков С. П. Критические точки и поверхности уровня многозначных функций // Тр. МИАН. 1984. Т. 166. С. 201-209.

[3] Кругликов Б. С. Точная гладкая классификация гамильтоновых векторных полей на двумерных многообразиях // Матем. заметки. 1997. Т. 61. № 2. С. 179-200.

[4] Болсинов А. В. Гладкая траекторная классификация интегрируемых гамильтоновых систем с двумя степенями свободы // Матем. сб. 1995. Т. 186. № 1. С. 3-28.

[5] Болсинов А. В., Фоменко А. Т. Траекторная эквивалентность интегрируемых гамильтоновых систем с двумя степенями свободы. Теорема классификации. I // Матем. сб. 1994. T. 185. № 4. С. 27-80; II // Матем. сб. 1994. Т. 185. № 5. С. 27-78.

[6] Болсинов А. В., Матвеев С. В., Фоменко А. Т. Топологическая классификация интегрируемых гамильтоновых систем с двумя степенями свободы. Список систем малой сложности // УМН. 1990. Т. 45. № 2. С. 59-77.

[7] Dufour J.-P., Molino P., Toulet A. Classification des systèmes intégrables en dimension 2 et invariants des modèles de Fomenko // C. R. Acad. Sci. Paris. Sér. I. Math. 1994. V. 318. № 10. P. 949-952.

[8] Colin de Verdiere Y., Vey J. Le lemme de Morse isochore // Topology. 1979. V. 18. P. 283-293.

[9] Арнольд В.И. Дополнительные главы теории обыкновенных дифференциальных уравнений. М.: Наука, 1978.

[10] Мозер Ю. Быстро сходящийся метод итераций и нелинейные дифференциальные уравнения // УМН. 1968. Т. 23. № 4. С. 179-238.

[11] Палис ЖК., ди Мелу В. Геометрическая теория динамических систем. Введение. М.: Мир, 1986.

Московский государственный технический университет им. Н. Э. Баумана 